\title{
Deregulation of a STAT3-Interleukin 8 Signaling Pathway Promotes Human Glioblastoma Cell Proliferation and Invasiveness
}

\author{
Núria de la Iglesia, ${ }^{1 \star}$ Genevieve Konopka, ${ }^{1,2 *}$ Kah-Leong Lim, ${ }^{1}$ Catherine L. Nutt, ${ }^{4}$ Jacqueline F. Bromberg, ${ }^{5}$ \\ David A. Frank, ${ }^{5}$ Paul S. Mischel, ${ }^{6}$ David N. Louis, ${ }^{4}$ and Azad Bonni ${ }^{1,2}$ \\ ${ }^{1}$ Department of Pathology, ${ }^{2}$ Program in Neuroscience, and ${ }^{3}$ Department of Medical Oncology, Dana-Farber Cancer Institute, Harvard Medical School, \\ Boston, Massachusetts 02115, ${ }^{4}$ Department of Pathology and Cancer Center, Massachusetts General Hospital and Harvard Medical School, Boston, \\ Massachusetts 02114, ${ }^{5}$ Department of Medicine, Memorial Sloan-Kettering Cancer Center, New York, New York 10021, and ${ }^{6}$ Department of Molecular and \\ Medical Pharmacology and Medicine, David Geffen School of Medicine at the University of California, Los Angeles, Los Angeles, California 90095
}

Inactivation of the tumor suppressor phosphatase and tensin homolog (mutated in multiple advanced cancers 1) (PTEN) is recognized as a major event in the pathogenesis of the brain tumor glioblastoma. However, the mechanisms by which PTEN loss specifically impacts the malignant behavior of glioblastoma cells, including their proliferation and propensity for invasiveness, remain poorly understood. Genetic studies suggest that the transcription factor signal transducers and activators of transcription 3 (STAT3) harbors a PTENregulated tumor suppressive function in mouse astrocytes. Here, we report that STAT3 plays a critical tumor suppressive role in PTENdeficient human glioblastoma cells. Endogenous STAT3 signaling is specifically inhibited in PTEN-deficient glioblastoma cells. Strikingly, reactivation of STAT3 in PTEN-deficient glioblastoma cells inhibits their proliferation, invasiveness, and ability to spread on myelin. We also identify the chemokine interleukin 8 (IL8) as a novel target gene of STAT3 in human glioblastoma cells. Activated STAT3 occupies the endogenous IL8 promoter and directly represses IL8 transcription. Consistent with these results, IL8 is upregulated in PTEN-deficient human glioblastoma tumors. Importantly, IL8 repression mediates STAT3 inhibition of glioblastoma cell proliferation, invasiveness, and spreading on myelin. Collectively, our findings uncover a novel link between STAT3 and IL8, the deregulation of which plays a key role in the malignant behavior of PTEN-deficient glioblastoma cells. These studies suggest that STAT3 activation or IL8 inhibition may have potential in patient-tailored treatment of PTEN-deficient brain tumors.

Key words: STAT3; IL8; astrocyte; astroglia; gliogenesis; glioma; tumor

\section{Introduction}

Malignant gliomas are the most common primary tumors of the brain and among the most aggressive human tumors (Holland, 2001; Konopka and Bonni, 2003; Louis, 2006; Furnari et al., 2007). Despite intense efforts to find effective treatments, malignant gliomas are still incurable. Glioblastoma tumor cells display phenotypic characteristics of astrocytes and their precursors, the neural stem cells (Louis et al., 2007). Both neural stem cells and astrocytes have been proposed to represent the cells of origin of

\footnotetext{
Received Dec. 5, 2007; revised April 9, 2008; accepted April 9, 2008.

This work was supported by awards from the Stewart Trust of Washington, DC (A.B.), the Armenise-Harvard Foundation (A.B.), and the Carolyn and Peter Lynch Research Fund (A.B.); National Institutes of Health (NIH) Grant R01 CA57683 (D.N.L.); NIH Grants NS051255, NS41021, and NS047188 (A.B.); and a postdoctoral fellowship from the Fundación Ramón Areces, Spain, and a Taplin Postdoctoral Fellowship (N.d.I.I.). A.B. is the recipient of a fellowship from the Alfred P. Sloan Foundation, the Robert H. Ebert Clinical Scholar Award from the Esther A. and Joseph Klingenstein Fund, the EJLB Foundation Award, and the Sidney Kimmel Foundation Award. We thank Judith Stegmüller for providing adult rat brain myelin and Jeng-Shin Lee, Andrew Keates, and Joan Brugge for providing plasmids.

*N.d.I.I. and G.K. contributed equally to this manuscript.

Correspondence should be addressed to Azad Bonni at the above address. E-mail: azad_bonni@hms.harvard.edu.

DOI:10.1523/JNEUROSCI.5385-07.2008

Copyright $\odot 2008$ Society for Neuroscience $\quad$ 0270-6474/08/285870-09\$15.00/0
}

glioblastoma (Holland, 2001; Bachoo et al., 2002; Uhrbom et al., 2002; Bajenaru et al., 2003). Collectively, these studies suggest that deregulation of mechanisms that control the normal biology of neural stem cells and astrocytes, including the differentiation of neural stem cells into astrocytes, might contribute to the pathogenesis of glioblastoma.

The transcription factor signal transducers and activators of transcription 3 (STAT3) plays an instructive role in astrocyte differentiation during normal brain development (Bonni et al., 1997; Rajan and McKay, 1998). These observations raised the possibility that STAT3 signaling might be deregulated in glioblastoma. In genetic studies in mouse astrocytes, we recently identified STAT3 as an important regulator of glial cell transformation (de la Iglesia et al., 2008). STAT3 harbors a tumor suppressive or oncogenic function depending on the genetic background of the tumor. STAT3 is required for astrocyte transformation after expression of the oncogenic form of the epidermal growth factor receptor (EGFR), EGFRvIII. Conversely, STAT3 displays tumor suppressive properties in astrocytes deficient in the tumor suppressor phosphatase and tensin homolog (mutated in multiple advanced cancers 1) (PTEN) (de la Iglesia et al., 2008). A tumor suppressive role of STAT3 is consistent with the function of 
STAT3 as a transcription factor dedicated to the differentiation of neural stem cells into astrocytes during normal brain development (Bonni, 2003).

Genetic studies in mouse astrocytes suggest that deregulation of the PTEN-Akt pathway triggers astrocyte transformation by inhibiting the leukemia inhibitory factor receptor $\beta$ (LIFR $\beta$ )STAT3 pathway (de la Iglesia et al., 2008). Consistent with these results, PTEN deficiency in human glioblastoma specimens correlates with LIFR $\beta$ downregulation and inhibition of STAT3 phosphorylation at the key regulatory site Tyr705 (de la Iglesia et al., 2008). However, the effect of manipulating STAT3 activity on the malignant behavior of glioblastoma tumor cells and the mechanism by which STAT3 might mediate its tumor suppressive effects in glioblastoma cells has remained unknown.

In this study, we establish a tumor suppressive function of STAT3 in human glioblastoma cells. Activation of endogenous STAT3 in wild-type PTEN-expressing glioblastoma cells inhibits cell proliferation. Conversely, expression of a constitutive active form of STAT3 in PTEN-deficient glioblastoma cells significantly inhibits cell proliferation, invasiveness, and spreading on myelin. We also report a mechanism by which STAT3 triggers tumor suppressive effects in human glioblastoma cells. The chemokine interleukin 8 (IL8) is identified as a direct repressed target gene of STAT3 in human glioblastoma cells. The repression of IL8 mediates the ability of STAT3 to inhibit glioblastoma cell proliferation, invasiveness, and spreading on myelin. Together, our results define the STAT3-IL8 signaling link as a novel PTEN-regulated mechanism that suppresses glioblastoma cell proliferation and invasiveness. These findings provide new targets for potential patient-tailored therapeutic intervention in glioblastoma. Activation of STAT3 and inhibition of IL8 might represent useful strategies for the treatment of PTEN-deficient malignant gliomas.

\section{Materials and Methods}

Cell culture. U87 glioblastoma cells were infected with MSCV-internal ribosomal entry site (IRES)-green fluorescent protein (GFP)/S3C, MSCV-IRES-GFP/mS3C, or empty vector retroviruses, and a population of infected cells for each construct was collected by fluorescenceactivated cell-sorting for GFP expression. IL8 knockdown U87 cells were obtained by infection with pLL3.7-Puro/IL8i1 or pLL3.7-Puro/IL8i2 lentiviruses and selection with puromycin $(4 \mu \mathrm{g} / \mathrm{ml})$.

Plasmids. The MSCV-IRES-GFP/mS3C construct was generated by site-directed mutagenesis of RcCMV/S3C (Bromberg et al., 1999) using the QuikChange Site-Directed Mutagenesis kit (Stratagene) and subcloning into MSCV-IRES-GFP.

Virus production and infection. Recombinant retroviruses were made by transfecting human embryonic kidney 293T (HEK293T) cells with pMD.MLV gag.pol, pHDM.G [vesicular stomatitis virus glycoprotein (VSVG)], and the transfer plasmid (e.g., MSCV-IRES-GFP). Cells were infected with equal amounts of retroviruses in the presence of $8 \mu \mathrm{g} / \mathrm{ml}$ polybrene (Sigma-Aldrich)

Cloning of recombinant lentiviruses coding for a short hairpin RNA directed against STAT3 or human IL8 was performed using a modified pLL3.7 vector, which encodes resistance to puromycin (pLL3.7 Puro). The following complementary oligonucleotides were inserted into pLL3.7 Puro: Stat3i forward, 5'-TGG GCA GTT TGA GTC GCT CAT TCA AGA GAT GAG CGA CTC AAA CTG CCC TTT TTG GAA AC-3', Stat3i reverse, 5' -TCG AGT TTC CAA AAA GGG CAG TTT GAG TCG CTC ATC TCT TGA ATG AGC GAC TCA AAC TGC CCA-3'; IL8i 1 forward, 5' -TGA ACT TAG ATG TCA GTG CAT TCA AGA GAT GCA CTG ACA TCT AAG TTC TTT TTG GAA AC-3', IL8i 1 reverse, 5' -TCG AGT TTC CAA AAA GAA CTT AGA TGT CAG TGC ATC TCT TGA ATG CAC TGA CAT CTA AGT TCA-3'; IL8i 2 forward, 5'-TGA ACT GAG AGT GAT TGA GAG TTC AAG AGA CTC TCA ATC ACT CTC
AGT TCT TTT TGG AAA C-3', IL8i 2 reverse, 5' -TCG AGT TTC CAA AAA GAA CTG AGA GTG ATT GAG AGT CTC TTG AAC TCT CAA TCA CTC TCA GTT CA-3'. Hairpin structures containing the stem sequences (underlined) and the loops (bold italics) are indicated. Lentiviruses were generated by cotransfecting pLL3.7 and packaging vectors (VSVG, RSV-REV, and pMDL g/p RRE) into HEK293T cells. Cells were infected with equal amounts of lentiviruses and selected with puromycin.

Chromatin immunoprecipitation. Chromatin immunoprecipitation analyses were done as described previously (Shi et al., 2003). After immunoprecipitation, a PCR was used to amplify the IL8 promoter with the following primers: IL8, first PCR, human IL8 forward, $5^{\prime}$-TCT CAC TCC ATC CCT TTT GC-3' , human IL8 reverse, 5' -AGT GGC AGG TGT TAG AAC AAG A-3', nested PCR, human IL8 nest forward, 5' -CTC CAT CCC TTT TGC TAG TGA-3', human IL8 nest reverse, 5' -ACA GAT GCT ATC ATG ATG GTG AA-3'. Primers designed to amplify the E-cadherin promoter were used as negative controls for the PCR: human E-cad forward, 5'-TAG CCT GGC GTG GTG GTG TGC ACC TG-3', human E-cad reverse, 5'-GTG CGT GGC TGC AGC CAG GTG AGC C-3'.

Matrigel invasion assays. Matrigel precoated invasion chambers (BD Biosciences) with an $8 \mu \mathrm{m}$ pore size membrane were used according to the manufacturer instructions. Cells $\left(2.5 \times 10^{4}\right)$ in $500 \mu \mathrm{l}$ of serum-free DMEM were added to each of the inserts and incubated at $37^{\circ} \mathrm{C}$ for $22 \mathrm{~h}$. Cells on the lower surface of the membrane, which had migrated through the matrigel, were fixed, stained with crystal violet, and counted. Noninvasive NIH3T3 cells were used as a negative control. Equivalent numbers of NIH3T3 failed to invade the matrigel.

Microarray analysis. RNA was extracted from U87 stable cell lines using Trizol, followed by an additional purification step using an RNAeasy kit (Qiagen) according to the manufacturer instructions. Biotinylated cRNAs from each cell line were generated from $15 \mu \mathrm{g}$ of total RNA and hybridized to the Affymetrix U133A chips. Microarray procedures were conducted at the Dana-Farber Cancer Institute Microarray Core Facility (Boston, MA) (http://chip.dfci.harvard.edu). Each cell line was used in three separate experiments. Gene expression data were analyzed using Vector Xpression software (InforMax). Raw expression values were normalized by linear scaling so that the mean array intensity was identical for all scans. Intensity thresholds were set at a minimum of 20 and maximum of 16,000 units, resulting in 12,284 probe sets for subsequent analysis. These remaining 12,284 probe sets were then subjected to the $t$ test using Vector Xpression for the identification of differentially expressed transcripts. Fold change expression data were diagrammatically represented using GeneCluster software (http://www.broad.mit.edu/cancer/ software/genecluster2/gc2.html).

\section{Results}

STAT3 suppresses human glioblastoma cell proliferation

The identification of the tumor suppressive function of STAT3 in genetic studies of PTEN-deficient mouse astrocytes raises the major question of the role of STAT3 in human glioblastoma. To investigate STAT3 signaling in human glioblastoma cells, we first characterized the potential of the cytokine LIF to induce the phosphorylation of endogenous STAT3 at the key regulatory site Tyr705 in PTEN-expressing and PTEN-deficient glioblastoma cells. We found that, although LIF induced STAT3 Tyr705 phosphorylation in the wild-type PTEN-expressing SF188 and LN229 glioblastoma cells, LIF failed to effectively induce the STAT3 phosphorylation in the PTEN-deficient U87 and A172 glioblastoma cells (Fig. 1A). These results suggest that PTEN deficiency suppresses the endogenous STAT3 signaling pathway in human glioblastoma cells.

PTEN deficiency suppresses STAT3 signaling in mouse astrocytes via downregulation of the cytokine receptor LIFR $\beta$ (de la Iglesia et al., 2008). We therefore analyzed the expression of LIFR $\beta$ in human glioblastoma cells. Consistent with results in mouse astrocytes (de la Iglesia et al., 2008), we found very low levels of LIFR $\beta$ protein in the PTEN-deficient U87 glioblastoma 
cells (supplemental Fig. 1, available at www. jneurosci.org as supplemental material). These results are consistent with the interpretation that LIFR $\beta$ downregulation may represent a mechanism by which PTEN deficiency suppresses STAT3 signaling in the U87 glioblastoma cells. Two lines of evidence suggested that inhibition of STAT3 signaling was specific to LIFR $\beta$ deficiency in U87 cells. First, treatment of U87 cells with the phosphatidyl inositol 3-kinase inhibitor LY294002 [2-(4-morpholinyl)-8phenyl-4H-1-benzopyran-4-one] restored LIFR $\beta$ levels (supplemental Fig. $2 A$, available at www.jneurosci.org as supplemental material). Second, reconstitution of LIFR $\beta$ signaling in U87 cells by expression of a chimeric protein consisting of the intracellular domain of LIFR $\beta$ fused to the ectodomain of EGFR allowed the induction of STAT3 phosphorylation at Tyr705 in response to EGF (supplemental Fig. 2B, available at www.jneurosci.org as supplemental material). These findings suggest that the absence of LIFR $\beta$ in the PTENdeficient U87 glioblastoma cells may underlie the inactivation of STAT3 signaling in these cells. These results corroborate our findings in murine astrocytes (de la Iglesia et al., 2008). However, as a caveat that not all human PTEN-deficient glioblastoma cells behave in this manner, we find that LIFR $\beta$ expression is intact in A172 cells (supplemental Fig. 1, available at www. jneurosci.org as supplemental material), suggesting that PTEN deficiency may inhibit STAT3 signaling independently of regulation of LIFR $\beta$ expression in these cells.

We also measured the levels of STAT3 phosphorylation at Ser727 in the human glioblastoma cells. These experiments revealed that STAT3 was phosphorylated at Ser727 in the human glioblastoma cells regardless of their PTEN status, and treatment with LIF had little or no effect on the serine 727 phosphorylation in these cells (supplemental Fig. 3, available at www. jneurosci.org as supplemental material).

These results suggest that the PTEN status of human glioblastoma cells predominantly affects the phosphorylation of STAT3 at Tyr705.

We next asked whether LIF had distinct effects on proliferation in glioblastoma cells in parallel with the differential phosphorylation of endogenous STAT3 at Tyr705 depending on the PTEN status of these cells. Remarkably, although LIF inhibited the proliferation of the PTEN-expressing SF188 and LN229 glioblastoma cells (Fig. $1 B-D$ ), LIF had little or no effect on the proliferation of the PTEN-deficient A172 and U87 glioblastoma cells (Fig. $1 \mathrm{~B}$ ), suggesting that LIF inhibition of cell proliferation correlates with LIF induction of endogenous STAT3 in PTENexpressing glioblastoma cells.

To determine the role of endogenous STAT3 activation in LIF
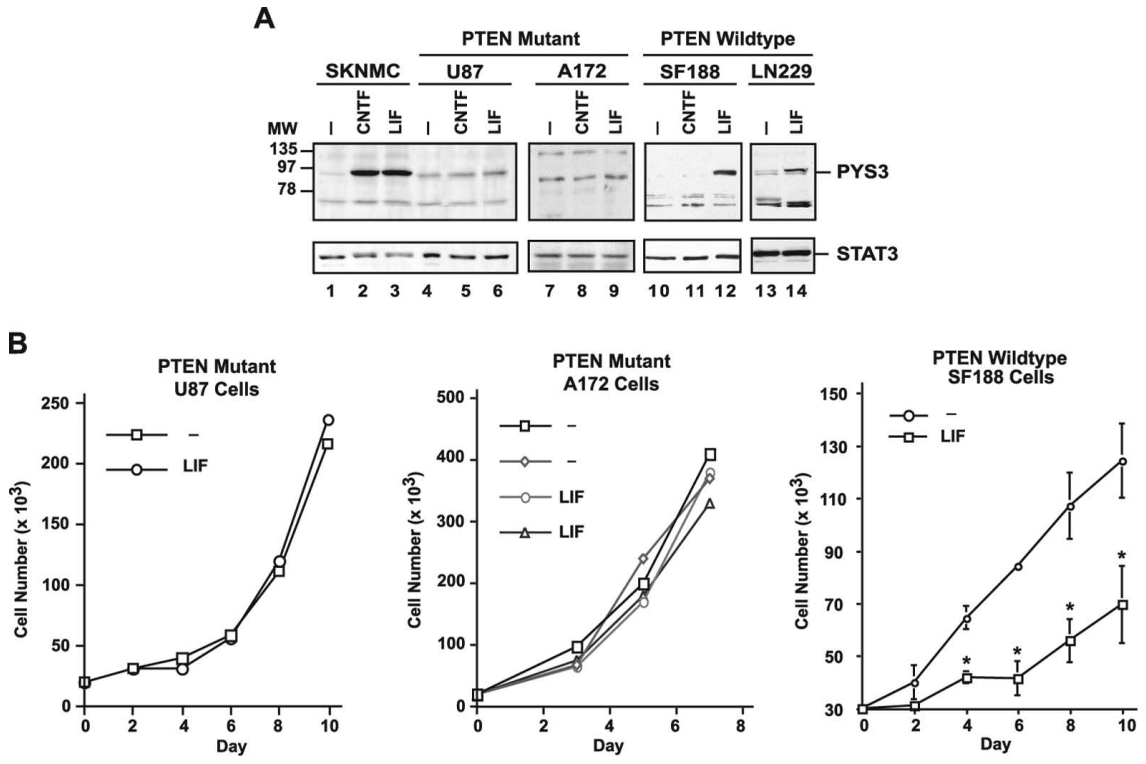

C SF188 Cells

D
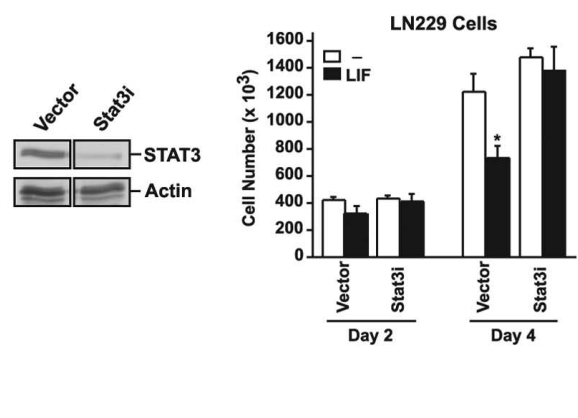

Figure 1. Endogenous STAT3 inhibits proliferation of wild-type PTEN-expressing glioblastoma cells. $A$, Lysates of glioblastoma cells that express wild-type PTEN (SF188 and LN229) or glioblastoma cells that harbor PTEN mutations (U87 and A172) were immunoblotted with STAT3 and Tyr 705-phosphorylated STAT3 (pYS3) antibodies. Cells were serum starved for $24 \mathrm{~h}$ and then KKNMC neuroblastoma cells and in the wild-type PTEN-expressing glioblastoma cells but failed to effectively induce the STAT3 sphorylation in the PTEN-deficient glioblastoma cells. B, Growth curves of PTEN-mutant (U87 and A172) and PTEN作 (S3D). LF $(10 \mathrm{ng} / \mathrm{ml}$ daily) led to a significant reduction in population growth of control vector or wild-type STAT3-expressing cells ( $n=3$; ANOVA, ${ }^{*} p<0.05,{ }^{n} p<0.01,{ }^{\S} p<0.001$ ) but not in S3D-expressing SF188 cells. D, Left, Lysates of LN229 cells infected with either control lentivirus or lentivirus encoding a small interfering hairpin RNA directed at STAT3 and selected with puromycin were immunoblotted with an STAT3 antibody. Actin was used as loading control. The Stat3i hairpin RNA induced ous human STAT3. Right, Time course of the growth of LN229 cells infected with control lentivirus or lentivirus encoding Stat $3 i$ and selected with puromycin. LIF ( $10 \mathrm{ng} / \mathrm{ml}$ daily) led to a significant reduction in growth in controlinfected $\left(n=3\right.$; ANOVA, $\left.{ }^{*} p<0.05\right)$ but not Stat3i hairpin RNA-expressing LN229 cells.

inhibition of cell proliferation in PTEN-expressing glioblastoma cells, we expressed in SF188 cells a dominant interfering form of STAT3 (S3D) that forms dimers with endogenous STAT3 that fail to bind to promoters of cytokine responsive genes (Nakajima et al., 1996; Bonni et al., 1997). We found that the expression of S3D, but not wild-type STAT3, significantly reduced the ability of LIF to inhibit SF188 glioblastoma population growth (Fig. 1C). We also used a lentiviral DNA-based template method of RNA interference to knockdown STAT3 protein in the PTENexpressing LN229 glioblastoma cells. STAT3 knockdown was found to significantly inhibit LIF suppression of proliferation in LN229 glioblastoma cells (Fig. 1D). In other experiments, STAT3 knockdown in SF188 glioblastoma cells also blocked LIF inhibition of SF188 cell proliferation (data not shown). Together, our 


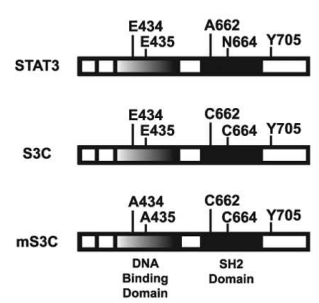

C

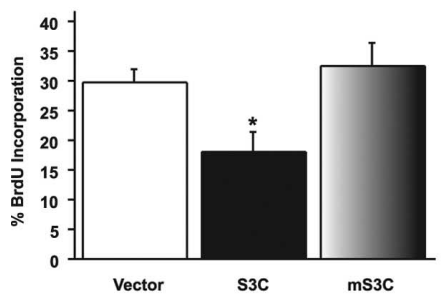

B
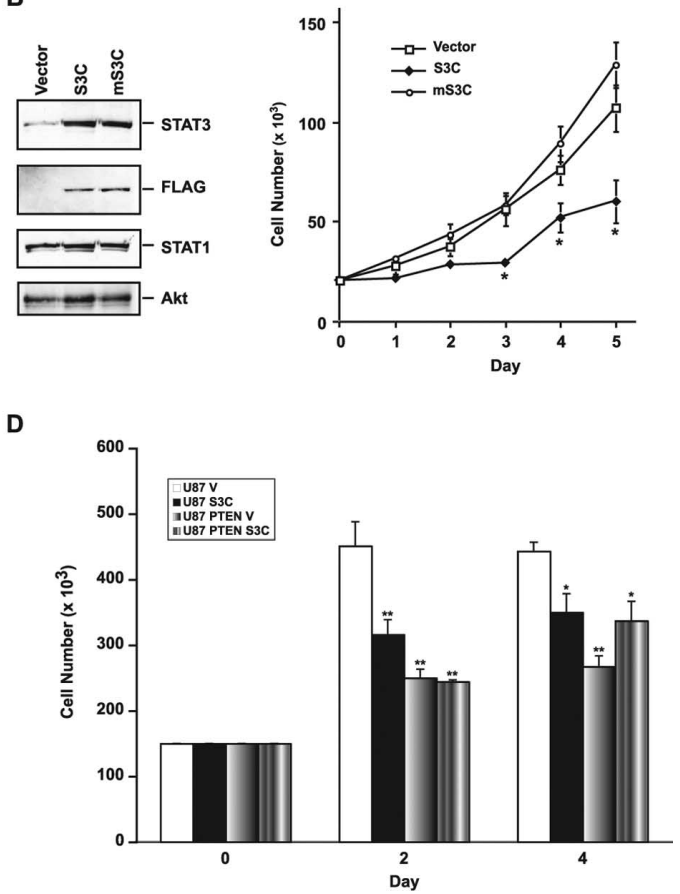

Figure 2. Expression of activated STAT3 inhibits proliferation PTEN-deficient glioblastoma cells. $\boldsymbol{A}$, Schematic of activated STAT3 (S3C) or a DNA-binding mutant version of S3C (mS3C). B, Left, Lysates of U87 glioblastoma cells infected with a control retrovirus containing an IRES-GFP cassette or retroviruses that also encode FLAG-tagged S3C or mS3C were immunoblotted with STAT3 and FLAG antibodies and antibodies to STAT1 or Akt as loading controls. Right, Cell population growth curves of U87 glioblastoma cells. S3C-expressing U87 cells proliferated significantly more slowly than mS3C-expressing U87 cells or those infected with control virus $\left(n=3\right.$; ANOVA $\left.{ }^{*} p<0.05\right)$. C, BrdU labeling of U87 glioblastoma stable cells measured as a percentage of the total number of cells. Incorporation of BrdU was significantly reduced in U87-S3C cells compared with both vector and $\mathrm{mS3C}\left(n=4\right.$; ANOVA, $\left.{ }^{*} p<0.05\right)$. $\boldsymbol{D}$, Cell population growth curves of PTEN-deficient U87 cells or isogenic PTENexpressing U87 cells infected with the $\mathrm{S3C}$ or control retrovirus. Expression of $\mathrm{S3C}$ significantly reduced the proliferation of U87 but not of isogenic PTEN-expressing U87 glioblastoma cells (representative experiment of 3 independent experiments performed in triplicate; ANOVA, $^{*} p<0.001,{ }^{* *} p<0.0001$ ).

results indicate that LIF induction of endogenous STAT3 in wildtype PTEN-expressing glioblastoma cells inhibits cell proliferation. These results are also consistent with the possibility that inhibition of endogenous STAT3 signaling after PTEN loss may serve to promote glioblastoma cell proliferation.

To directly assess the significance of PTEN loss suppression of STAT3 signaling in the malignant behavior of glioblastoma cells, we determined the effect of reactivation of STAT3 in PTENdeficient glioblastoma cells. Using a retroviral approach, we expressed a constitutively active form of STAT3 (S3C) in these cells. S3C contains two point mutations whereby A661 and N663 are replaced with cysteine. S3C thus forms dimers through sulfhydryl bonds independently of Tyr705 phosphorylation and translocates to the nucleus independently of cytokine stimulation (Bromberg et al., 1999). We generated a mutant form of activated STAT3 (S3C) in which the DNA binding region of S3C was mutated (mS3C) (Fig. 2A). When expressed in HEK293T cells, activated STAT3 (S3C), but not the mutant form $\mathrm{mS3C}$, robustly induced transcription of a reporter gene driven by STAT binding sites, suggesting that $\mathrm{mS} 3 \mathrm{C}$ serves as a transcriptionally impaired control mutant of S3C (supplemental Fig. 4, available at www. jneurosci.org as supplemental material).

We stably expressed activated STAT3 (S3C) or its mutant form $\mathrm{mS3C}$ in PTEN-deficient U87 glioblastoma cells. In cell growth assays, activated STAT3 (S3C)- but not mS3C-expressing U87 glioblastoma cells increased in number at a significantly lower rate than control vector-infected U87 cells (Fig. 2 B). There

was $<1 \%$ cell death in control vectorinfected U87 glioblastoma cells, and the expression of activated STAT3 (S3C) or $\mathrm{mS3C}$ had no effect on cell death in the U87 cells (data not shown). In contrast, the rate of bromodeoxyuridine (BrdU) incorporation in activated STAT3 (S3C)- but not mS3C-expressing glioblastoma cells was reduced when compared with control vector-infected cells (Fig. 2C). These results suggest that activated STAT3 inhibits the proliferation of PTEN-deficient glioblastoma cells. In other cell population growth experiments, we found that exogenous expression of PTEN in the PTENdeficient U87 cells reduced the capacity of these cells to proliferate (Fig. 2D). In addition, whereas expression of $\mathrm{S} 3 \mathrm{C}$ reduced the proliferative capacity of the parental U87 cells, S3C failed to effectively reduce the proliferation of the isogenic PTENexpressing U87 cells (Fig. 2D). These results suggest that reactivation of STAT3 specifically inhibits the ability of PTEN deficiency to promote the proliferation of human glioblastoma cells.

\section{STAT3 suppresses glioblastoma cell invasiveness and spreading on myelin} Having established STAT3 as a suppressor of glioblastoma cell proliferation, we considered the possibility that STAT3 might also control glioblastoma cell invasiveness. We determined whether reactivation of STAT3 in the PTEN-deficient U87 glioblastoma cells alters their invasiveness. In matrigel invasion assays, U87 cells efficiently invaded the matrigel layer (Fig. 3A). However, expression of activated STAT3 (S3C) in U87 glioblastoma cells significantly reduced the ability of these cells to invade matrigel (Fig. 3A). STAT3 inhibition of U87 glioblastoma cell invasiveness was not secondary to the effect of activated STAT3 on cell proliferation, because the invasive potential of these cells was measured at a time ( $22 \mathrm{~h}$ after plating) before the inhibitory effects of activated STAT3 on U87 cell proliferation were manifest (Fig. 2B). These results suggest that reactivation of STAT3 suppresses the invasive property of PTENdeficient glioblastoma cells.

To determine whether STAT3 regulates glioblastoma cell behavior on a substrate that is relevant to the selective property of gliomas to invade brain tissue along white matter tracts, we tested the effect of activated STAT3 (S3C) on the ability of glioblastoma cells to adhere and spread on adult rat brain myelin (Amberger et al., 1998). Unlike NIH3T3 cells, which spread on polynornithine but not on myelin (data not shown), U87 glioblastoma cells spread equally well on polyornithine or myelin substrate forming actin stress fibers around the cell cortex (Fig. 3B). The selective ability of glioblastoma cells but not other tumor or immortalized cells to spread on myelin substrate provides a powerful assay that may reflect the propensity of malignant gliomas to migrate along brain white matter tracts (Amberger et al., 1998). Strikingly, in contrast to parental U87 glioblastoma cells or those infected with the control GFP virus or the $\mathrm{mS} 3 \mathrm{C}$ virus, the majority of S3Cexpressing U87 glioblastoma cells spread on polyornithine sub- 
A

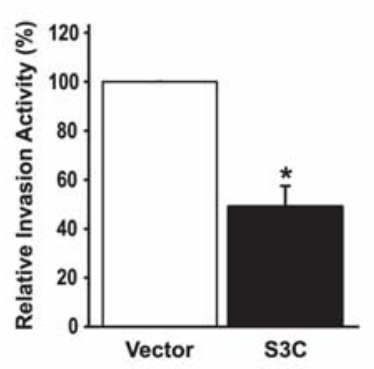

B

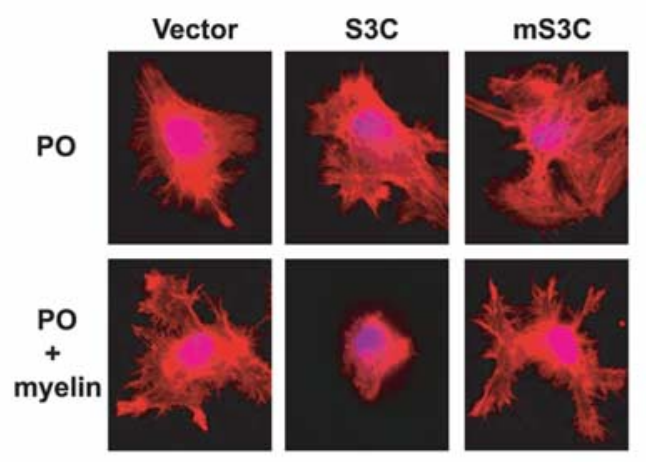

C

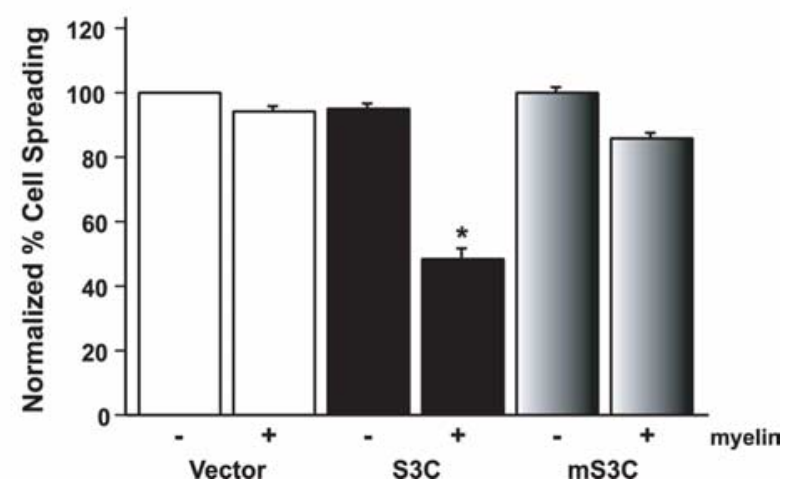

Figure 3. STAT3 decreases glioblastoma cell invasiveness and spreading on myelin. $A$, Matrigel invasion assay of U87 glioblastoma stable cell lines. Significantly more U87-vector cells invaded the matrigel substrate than U87-S3C cells $\left(n=3 ; t\right.$ test, $\left.{ }^{*} p<0.05\right)$. STAT3 inhibition of U87 glioblastoma cell invasiveness was not secondary to the effect of activated STAT 3 on U87 cell proliferation, because the invasive potential of these cells was measured at a time ( $22 \mathrm{~h}$ after plating) before the inhibitory effects of activated STAT3 on U87 cell proliferation (Fig. 2B). Equivalent numbers of NIH3T3 cells failed to invade the matrigel (data not shown). $\boldsymbol{B}$, Phalloidin red staining of actin stress fibers of stable U87 glioblastoma cells plated onto coverslips coated with either polyornithine (P0) or polyornithine together with myelin (20 $\mu \mathrm{g} / \mathrm{ml})$. U87-S3C glioblastoma cells failed to spread on myelin (middle bottom panel), compared with a spread appearance on a polyornithine control substrate (middle top panel). U87-vector and U87-mS3C glioblastoma cells spread and formed stress fibers on myelin (right and left bottom panels). C, Quantification of cell spreading of U87 stable cells on myelin. Significantly fewer U87-S3C glioblastoma cells spread on myelin compared with U87-vector glioblastoma cells ( $n=3$; ANOVA, ${ }^{*} p<0.0001$ ). Cells were counted in a blinded manner in three independent experiments, and the percentage of spreading was determined by calculating the number of spread cells over the total number of cells.

strate but failed to spread or form stress fibers in their periphery on myelin (Fig. $3 B, C$ ). Thus, activated STAT3 specifically inhibits glioblastoma cell adhesion and spreading on myelin.

STAT3 suppresses glioblastoma cell proliferation and invasiveness via repression of the chemokine IL8

Having identified the functional effects of STAT3 on glioblastoma cell proliferation and invasiveness, we next set out to eluci- date the mechanism by which STAT3 mediates these biological effects. Because the ability of STAT3 to inhibit glioblastoma cell proliferation and invasiveness correlated with its function as a transcription factor, we reasoned that activated STAT3 triggers changes in the expression of specific genes that mediate the glioblastoma suppressive properties of STAT3. We therefore subjected the activated STAT3 (S3C)-expressing and control GFPexpressing U87 glioblastoma cells to microarray analyses using Affymetrix chips. In these analyses, the most profound alteration was a fivefold repression of the gene encoding IL8 in S3Cexpressing cells compared with control cells (Fig. 4A,B).

IL8 is a chemokine that regulates neutrophil activation and chemoattraction (Baggiolini et al., 1994). In addition, IL8 has been implicated in the promotion of angiogenesis in a variety of tumors, including gliomas (Heidemann et al., 2003; Garkavtsev et al., 2004). Consistent with a role for IL8 in glioblastoma progression, although IL8 expression is very low in normal brain, IL8 is upregulated in gliomas and in particular in high-grade astrocytoma and glioblastoma (Yamanaka et al., 1994). In a panel of human glioblastoma tumors we characterized by immunoblotting, we found that eight of 25 (32\%) tumors displayed high levels of IL8 (Fig. 4C). PTEN deficiency tightly correlates with inactivation of STAT3 signaling in these human glioblastoma samples (de la Iglesia et al., 2008). Importantly, the majority of the IL8expressing tumors (six of eight) had low PTEN levels (Fig. 4C) [for PTEN levels, see the study by de la Iglesia et al. (2008), their Fig. 6]. Fisher's exact test of IL8-positive and IL8-negative tumors revealed a strong correlation of IL8 expression with low PTEN levels (Fig. 4C). Together, these observations raised the question of whether the repression of IL8 mediates STAT3 inhibition of glioblastoma cell proliferation and invasiveness.

First, we confirmed the microarray data in Northern analyses in which the amount of IL8 mRNA was robustly suppressed in activated STAT3 (S3C)-expressing cells (Fig. 4D). Consistent with these results, immunoblotting of U87 glioblastoma cell extracts revealed that IL8 protein was significantly downregulated after expression of activated STAT3 (S3C). In contrast, IL8 protein was reduced to a lesser extent in $\mathrm{mS} 3 \mathrm{C}$-expressing cells, suggesting that the transcriptional function of STAT3 is required for regulation of IL8 expression (Fig. 4D). The secretion of mature soluble IL8 into the medium by these glioblastoma cells correlated tightly with the results of Northern and Western blot analyses (Fig. 4D). Together, these results established that STAT3 inhibits the expression of IL8 in glioblastoma cells.

In transient expression assays, expression of activated STAT3 (S3C) potently diminished IL8 promoter-mediated transcription (Fig. 4E). By chromatin immunoprecipitation analyses of activated STAT3 (S3C)-expressing U87 glioblastoma cells, activated STAT3 was specifically bound to the endogenous IL8 promoter in these cells (Fig. 4F). These findings suggest that IL8 is a direct gene target of STAT3 and that activated STAT3 represses IL8 transcription.

To investigate the mechanism by which STAT3 represses IL8 gene expression, we determined the region of the IL8 promoter necessary for activated STAT3 to inhibit IL8 transcription. Deletion analyses of the IL8 promoter in transient expression assays revealed that activated STAT3 (S3C) repressed a reporter gene controlled by a promoter fragment containing $173 \mathrm{nt}$ upstream of the IL8 transcriptional start site (supplemental Fig. 5A, available at www.jneurosci.org as supplemental material). The -173 IL8 promoter fragment contains the nuclear factor $\kappa \mathrm{B}(\mathrm{NFkB})$ response element that is critical for the expression of IL8 in diverse biological settings, including malignant gliomas (Mukaida et al., 
A

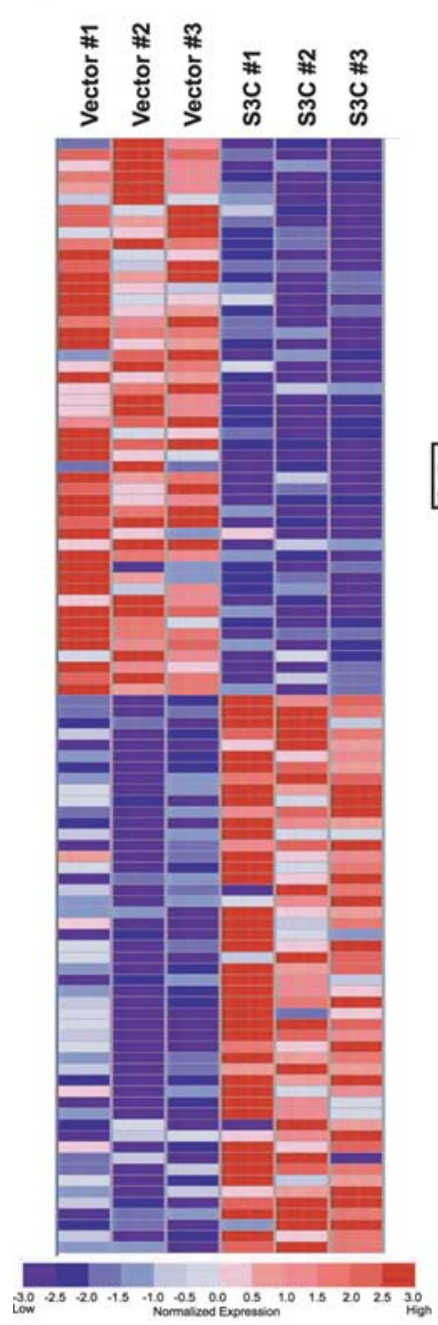

B

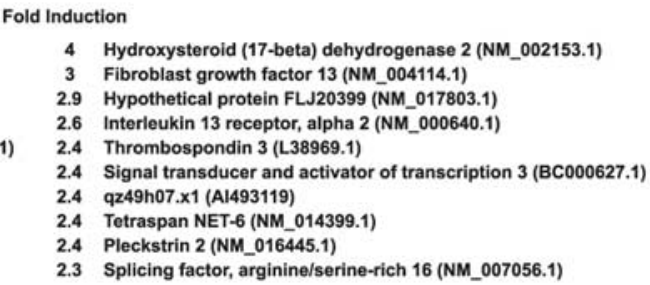

C $\begin{array}{lllllllllllllllllllllll}4 & 5 & 6 & 7 & 8 & 9 & 10 & 11 & 12 & 13 & 14 & 15 & 16 & 17 & 18 & 19 & 20 & 21 & 22 & 23 & 24 & 25 & \text { Number }\end{array}$

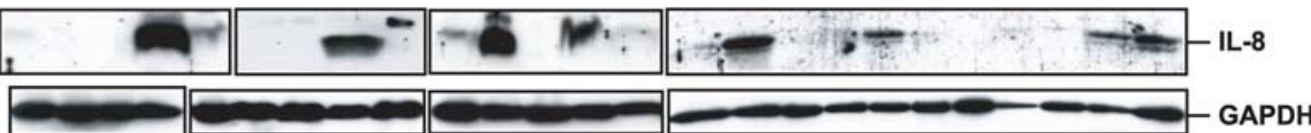

Human GBMs

\begin{tabular}{|c|c|c|}
\hline$p<0.01$ & low PTEN & high PTEN \\
\hline high IL8 & 6 & 2 \\
\hline low IL8 & 3 & 14 \\
\hline
\end{tabular}

D

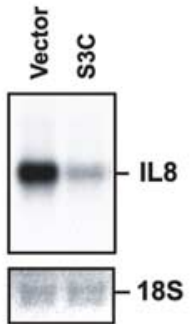

Northern

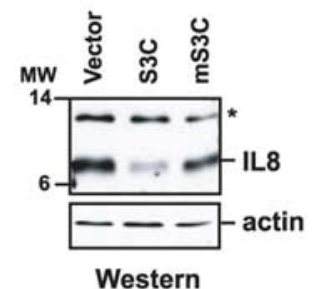

Western
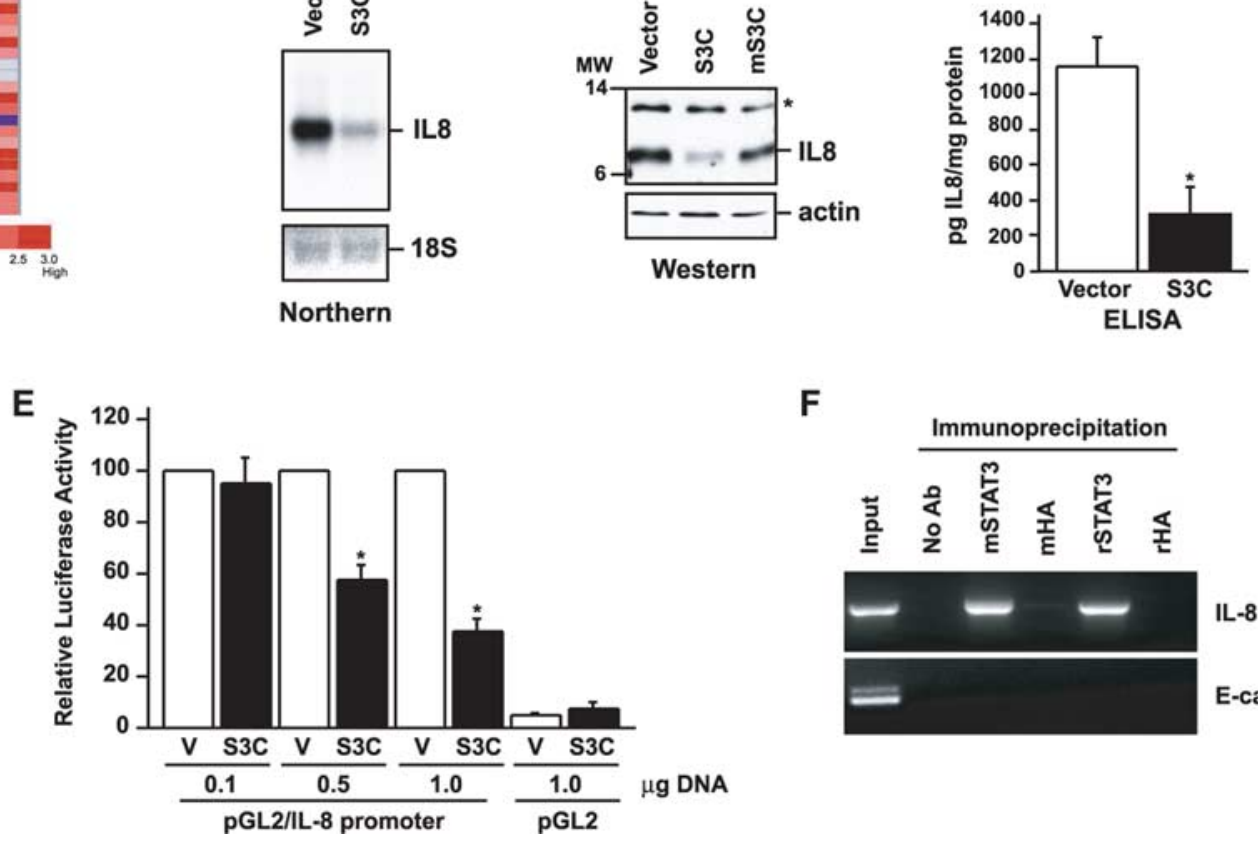

$\mathbf{F}$

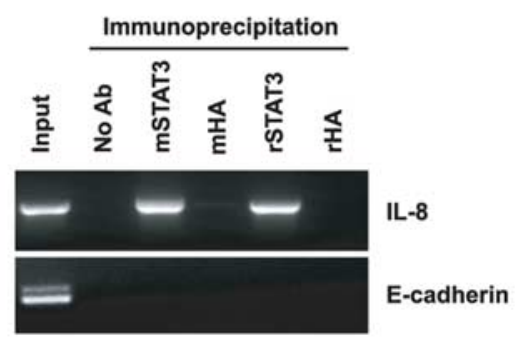

Figure 4. IL8 is a directSTAT3-repressed target gene. $A$, Diagrammatic representation of the top 50 genes in three independent microarray analyses of control or S3C-expressing U87 cells, ranked according to fold change, that were repressed or induced after S3C expression. B, Top 10 repressed (left) or induced (right) genes after S3C expression. The fold change is indicated, and GenBank numbers are in parenthesis. C, Top, Immunoblotting of lysates of human glioblastoma samples (GBM) with an IL8 antibody. Glyceraldehyde-3-phosphate dehydrogenase (GAPDH) was used as loading control [GAPDH immunoblotting of some of the GBM samples is also shown by de la Iglesia et al. (2008)]. Eight of the tumors $(\# 4,8,11,13,16,19,24$, and 25$)$ displayed very high levels of IL8. The majority of these tumors (6 of 8) also had low PTEN levels (de la Iglesia et al., 2008). Bottom, Fisher's exact test of IL8-positive and IL8-negative tumors revealed a strong correlation between IL8 expression and low PTEN levels [odds ratio (OR), 14; $p<0.01$ ]. D, Left, Northern analysis of control vector-infected U87 glioblastoma cells or those expressing S3C using an IL8 probe. 18S RNA levels indicate equal loading. Middle, Immunoblotting of lysates of control U87 glioblastoma cells or those expressing S3C or mS3C with an IL8 antibody. The asterisk indicates a nonspecific band. Right, Sandwich ELISA chemiluminescent analysis of medium from the control U87 glioblastoma cells or those expressing S3 C using two antibodies specific for human IL8 (R\&D Systems). Shown are mean IL8 concentration \pm SEM (pg of IL $8 / \mathrm{mg}$ of cell protein; $n=3 ; t$ test, ${ }^{*} p<0.01$ ). E, STAT3 represses the IL8 promoter. Pten ${ }^{-1-}$ astrocytes were transfected with increasing amounts of an expression plasmid encoding S3C or a control plasmid together with an IL 8 promoter-controlled luciferase reporter gene and a renilla expression plasmid and subjected to dual luciferase assay $48 \mathrm{~h}$ after transfection. Activated STAT3 significantly reduced IL8 promoter activity ( $n=3$; ANOVA, $\left.{ }^{*} p<0.0001\right)$. V, Vector. $F$, Chromatin immunoprecipitation analysis at the endogenous IL8 promoter in S3C-expressing U87 glioblastoma cells using two different STAT3 antibodies ( $\mathrm{m}$, mouse; $r$, rabbit). Mouse and rabbit HA antibodies were used as controls. Negative controls for the PCR were performed with primers for the E-cadherin gene. STAT3 directly bound to the endogenous IL8 gene. 
1990; Brat et al., 2005; Sarkar et al., 2008). We therefore asked whether activated STAT3 might inhibit NFkB-dependent transcription in glial cells. Expression of activated STAT3 (S3C) inhibited a luciferase reporter gene controlled by multiple NFkB-binding sites (supplemental Fig. 5B, available at www.jneurosci.org as supplemental material). Together, these results suggest that activated STAT3 may repress the IL8 gene via inhibition of NFkB.

To determine whether IL8 repression contributes to the ability of STAT3 to inhibit glioblastoma cell proliferation, we used a lentiviral DNA template-based method of RNA interference to knockdown IL8 in U87 glioblastoma cells (Fig. $5 A)$. Knockdown of endogenous IL8 in U87 glioblastoma cells by either of two hairpin RNAs targeting distinct regions of IL8 significantly reduced cell proliferation (Fig. 5B). IL8 knockdown also significantly reduced the ability of U87 glioblastoma cells to invade matrigel (Fig. $5 C$ ). In other experiments, we asked whether IL8 restores the ability of activated STAT3 (S3C)expressing U87 glioblastoma cells to spread on myelin substrate. After exposure to IL8, the S3C-expressing U87 glioblastoma cells spread as efficiently as control vectorinfected U87 cells on myelin (Fig. 5D). Together, these results suggest that IL8 is a STAT3-repressed gene that promotes glioblastoma cell proliferation and invasiveness (Fig. 5E).

\section{Discussion}

In this study, we uncovered a novel STAT3dependent tumor suppressive mechanism in human glioblastoma cells (see model in Fig. $5 E$ ). We found that cytokine-induced endogenous STAT3 signaling was specifically impaired in PTEN-deficient but not PTEN-expressing glioblastoma cells. Inhibition of endogenous STAT3 blocked cytokine suppression of proliferation in PTENexpressing glioblastoma cells. Conversely, reactivation of STAT3 in PTEN-deficient glioblastoma cells inhibited their proliferation, invasiveness, and spreading on myelin. We also identified the chemokine IL8 as a direct repressed target gene of STAT3 in glioblastoma cells. IL8 repression mediated the ability of STAT3 to inhibit glioblastoma cell proliferation and invasiveness. Therefore, deregulation of the STAT3-IL8 signaling link may confer PTEN loss with the ability to stimulate glioblastoma cell proliferation and invasiveness.

A major conclusion of our study is that STAT3, a protein with oncogenic behavior outside the brain (Bromberg et al., 1999; Bowman et al., 2000), exerts tumor suppressor effects in human glioblastoma cells. This finding provides pathophysiological significance to our recent studies indicating that STAT3 suppresses malignant transformation of mouse astrocytes (de la Iglesia et al., 2008). In some reports, it has been suggested that STATs may promote human glioblastoma tumor cell proliferation and sur-
B

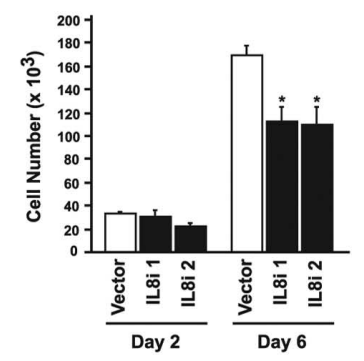

D

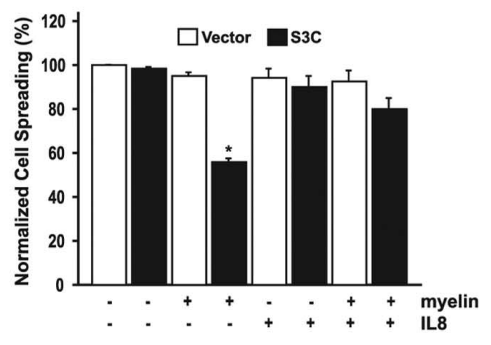

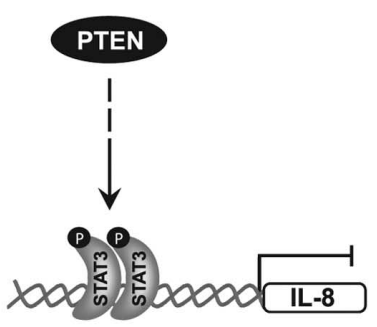

Glioblastoma cell proliferation and invasiveness

Figure 5. IL8 promotes glioblastoma cell proliferation, invasiveness, and spreading on myelin. $A$, Lysates of U87 cells infected with IL8 RNAi-encoding lentiviruses (IL8i 1 or IL8i 2) or an empty vector and selected with puromycin were immunoblotted with IL8 and actin antibodies. The asterisk indicates a nonspecific band. $\boldsymbol{B}$, Cell population growth curves of IL8 knockdown or

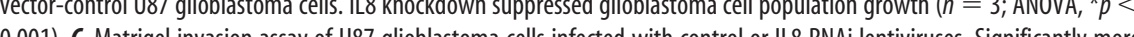
U87-vector glioblastoma cells invaded the matrigel substrate than U87-IL8i 1 or U87-IL8i 2 glioblastoma cells (ANOVA, ${ }^{*} p<$ 0.01). D, IL8 addition rescues STAT3 suppression of glioma cell spreading on myelin. Quantification of cell spreading shows that, in the presence of IL8, significantly more U87-S3C cells spread on myelin compared with untreated U87-S3C cells plated on myelin expression. Right, STAT3 is inhibited after PTEN deficiency, thus relieving repression of the IL8 gene. Upregulation of IL8 promotes glioblastoma cell proliferation and invasiveness.

vival (Rahaman et al., 2002; Konnikova et al., 2003), and STAT3 activation has been reported in human glial tumors (Weissenberger et al., 2004). On the other hand, other studies have provided evidence of the absence of STAT3 activation in human gliomas (Wang et al., 2004). We recently used a genetic approach in mouse astrocytes to directly assess STAT3 function in glial cell transformation. We found that the predominant role of STAT3 is to suppress malignant glial transformation, including in the context of PTEN deficiency (de la Iglesia et al., 2008). In agreement with these mouse genetic studies, our present findings reveal that STAT3 suppresses the malignant properties of PTEN-deficient glioblastoma cells, including their proliferation and invasiveness. Interestingly, expression of the glioblastoma-associated protein EGFRvIII in mouse astrocytes triggers an oncogenic switch in STAT3 function (de la Iglesia et al., 2008). In human glioblastomas, EGFRvIII expression correlates with STAT3 activation in both immunoblotting and immunohistochemical analyses (Mizoguchi et al., 2006; de la Iglesia et al., 2008). Therefore, it will 
be interesting to determine in future studies whether and how STAT3 might promote the malignant behavior of EGFRvIIIexpressing human glioblastoma cells.

Inhibition of the gliogenesis-promoting STAT3 signaling pathway in glioblastoma cells may serve to couple loss of a general tumor suppressor such as PTEN to the glioblastoma cell-specific behavior of invasiveness and adhesion and spreading on myelin. The PTEN-loss-triggered suppression of STAT3 signaling in glioblastoma cells illustrates how interactions between ubiquitously acting tumor suppressors and developmental signaling pathways implicated in cell differentiation and fate specification may produce tumor-specific pathological behaviors.

The identification of IL8 as a novel STAT3-repressed target gene in glioma cells, the repression of which mediates STAT3 inhibition of glioma cell proliferation, invasiveness, and spreading on myelin defines a novel cytokine-chemokine connection (Fig. 5E). Although STAT3 is commonly known to induce transcription (Darnell, 1997; Levy, 2003), our findings suggest that STAT3 may also act as a transcriptional repressor in glial cells. STAT3 appears to repress the IL8 promoter via inhibition of the $\mathrm{NFkB}$ response element. Interestingly, STAT3 inhibits expression of the inducible nitric oxide synthase (iNOS) gene in endothelial cells by directly inhibiting NFkB (Yu et al., 2002; Yu and Kone, 2004). The DNA binding domain of STAT3 directly interacts with NFkB and thereby inhibits NFkB-dependent iNOS transcription (Yu and Kone, 2004). These results are consistent with the finding that the DNA-binding domain of STAT3 is required for the ability of STAT3 to repress IL8 expression in glioblastoma cells (Fig. 4D). The candidate glial tumor suppressor inhibitor of growth 4 (ING4) reportedly inhibits glioblastoma-induced angiogenesis via repression of IL8, whereby ING4 inhibits NFkBdependent IL8 transcription (Garkavtsev et al., 2004). Collectively, these observations raise the possibility that STAT3 and ING4 may cooperate at the IL8 promoter to inhibit NFkB and thereby repress IL8 gene expression and thus control glioblastoma cell proliferation, invasiveness, and glioblastoma-induced angiogenesis.

The elucidation of STAT3 and IL8 in this study as critical regulators of glioblastoma cell proliferation and invasiveness provides new targets for therapeutic intervention. The chemokine IL8 and its cell surface receptors CXCR1 and CXCR2 are particularly attractive as potential targets in the treatment of PTEN-deficient glioblastoma. These chemokine receptors have been studied in the context of acute inflammation (Rollins, 1997; Schraufstatter et al., 2001), and small molecule inhibitors of CXCR1 and CXCR2 have been characterized (White et al., 1998; Bertini et al., 2004). It will be important to determine the potential of CXCR1 and CXCR2 antagonists to inhibit the malignant features of PTEN-deficient glioblastoma cells. These studies may provide the foundation for patient-tailored therapy in the management of brain tumors.

\section{References}

Amberger VR, Hensel T, Ogata N, Schwab ME (1998) Spreading and migration of human glioma and rat C6 cells on central nervous system myelin in vitro is correlated with tumor malignancy and involves a metalloproteolytic activity. Cancer Res 58:149-158.

Bachoo RM, Maher EA, Ligon KL, Sharpless NE, Chan SS, You MJ, Tang Y, DeFrances J, Stover E, Weissleder R, Rowitch DH, Louis DN, DePinho RA (2002) Epidermal growth factor receptor and Ink4a/Arf. Convergent mechanisms governing terminal differentiation and transformation along the neural stem cell to astrocyte axis. Cancer Cell 1:269-277.

Baggiolini M, Dewald B, Moser B (1994) Interleukin-8 and related chemotactic cytokines-CXC and CC chemokines. Adv Immunol 55:97-179.
Bajenaru ML, Hernandez MR, Perry A, Zhu Y, Parada LF, Garbow JR, Gutmann DH (2003) Optic nerve glioma in mice requires astrocyte Nf1 gene inactivation and Nf1 brain heterozygosity. Cancer Res 63:8573-8577.

Bertini R, Allegretti M, Bizzarri C, Moriconi A, Locati M, Zampella G, Cervellera MN, Di Cioccio V, Cesta MC, Galliera E, Martinez FO, Di Bitondo R, Troiani G, Sabbatini V, D’Anniballe G, Anacardio R, Cutrin JC, Cavalieri B, Mainiero F, Strippoli R, et al. (2004) Noncompetitive allosteric inhibitors of the inflammatory chemokine receptors CXCR1 and CXCR2: prevention of reperfusion injury. Proc Natl Acad Sci USA 101:11791-11796.

Bonni A (2003) STATs in the central nervous system. In: Signal transducers and activators of transcription (STATs). Activation and biology (Sehgal PB, Levy DE, Hirano T, eds), pp 663-685. Dordrecht, The Netherlands: Kluwer Academic.

Bonni A, Sun Y, Nadal-Vicens M, Bhatt A, Frank DA, Rozovsky I, Stahl N, Yancopoulos GD, Greenberg ME (1997) Regulation of gliogenesis in the central nervous system by the JAK-STAT signaling pathway. Science 278:477-483.

Bowman T, Garcia R, Turkson J, Jove R (2000) STATs in oncogenesis. Oncogene 19:2474-2488.

Brat DJ, Bellail AC, Van Meir EG (2005) The role of interleukin-8 and its receptors in gliomagenesis and tumoral angiogenesis. Neuro Oncol 7:122-133.

Bromberg JF, Wrzeszczynska MH, Devgan G, Zhao Y, Pestell RG, Albanese C, Darnell Jr JE (1999) Stat3 as an oncogene. Cell 98:295-303.

Darnell Jr JE (1997) STATs and gene regulation. Science 277:1630-1635.

de la Iglesia N, Konopka G, Puram SV, Chan JA, Bachoo RM, You MJ, Levy DE, Depinho RA, Bonni A (2008) Identification of a PTEN-regulated STAT3 brain tumor suppressor pathway. Genes Dev 22:449-462.

Furnari FB, Fenton T, Bachoo RM, Mukasa A, Stommel JM, Stegh A, Hahn WC, Ligon KL, Louis DN, Brennan C, Chin L, DePinho RA, Cavenee WK (2007) Malignant astrocytic glioma: genetics, biology, and paths to treatment. Genes Dev 21:2683-2710.

Garkavtsev I, Kozin SV, Chernova O, Xu L, Winkler F, Brown E, Barnett GH, Jain RK (2004) The candidate tumour suppressor protein ING4 regulates brain tumour growth and angiogenesis. Nature 428:328-332.

Heidemann J, Ogawa H, Dwinell MB, Rafiee P, Maaser C, Gockel HR, Otterson MF, Ota DM, Lugering N, Domschke W, Binion DG (2003) Angiogenic effects of interleukin 8 (CXCL8) in human intestinal microvascular endothelial cells are mediated by CXCR2. J Biol Chem 278:8508-8515.

Holland EC (2001) Gliomagenesis: genetic alterations and mouse models. Nat Rev Genet 2:120-129.

Konnikova L, Kotecki M, Kruger MM, Cochran BH (2003) Knockdown of STAT3 expression by RNAi induces apoptosis in astrocytoma cells. BMC Cancer 3:23.

Konopka G, Bonni A (2003) Signaling pathways regulating gliomagenesis. Curr Mol Med 3:73-84.

Levy DE (2003) STAT transcriptional activation mechanisms. In: Signal transducers and activators of transcription (STATs). Activation and biology (Sehgal PB, Levy DE, Hirano T, eds), pp 327-341. Dordrecht, The Netherlands: Kluwer Academic.

Louis DN (2006) Molecular pathology of malignant gliomas. Annu Rev Pathol 1:97-117.

Louis DN, Ohgaki H, Wiestler OD, Cavenee WK (2007) World Health Organization histological classification of tumours of the central nervous system. Lyon, France: IARC.

Mizoguchi M, Betensky RA, Batchelor TT, Bernay DC, Louis DN, Nutt CL (2006) Activation of STAT3, MAPK, and AKT in malignant astrocytic gliomas: correlation with EGFR status, tumor grade, and survival. J Neuropathol Exp Neurol 65:1181-1188.

Mukaida N, Mahe Y, Matsushima K (1990) Cooperative interaction of nuclear factor-kappa B- and cis-regulatory enhancer binding protein-like factor binding elements in activating the interleukin- 8 gene by proinflammatory cytokines. J Biol Chem 265:21128-21133.

Nakajima K, Yamanaka Y, Nakae K, Kojima H, Ichiba M, Kiuchi N, Kitaoka T, Fukada T, Hibi M, Hirano T (1996) A central role for Stat3 in IL-6induced regulation of growth and differentiation in M1 leukemia cells. EMBO J 15:3651-3658.

Rahaman SO, Harbor PC, Chernova O, Barnett GH, Vogelbaum MA, Haque SJ (2002) Inhibition of constitutively active Stat3 suppresses proliferation and induces apoptosis in glioblastoma multiforme cells. Oncogene 21:8404-8413. 
Rajan P, McKay RD (1998) Multiple routes to astrocytic differentiation in the CNS. J Neurosci 18:3620-3629.

Rollins BJ (1997) Chemokines. Blood 90:909-928.

Sarkar D, Park ES, Emdad L, Lee SG, Su ZZ, Fisher PB (2008) Molecular basis of nuclear factor-kappaB activation by astrocyte elevated gene-1. Cancer Res 68:1478-1484.

Schraufstatter IU, Chung J, Burger M (2001) IL-8 activates endothelial cell CXCR1 and CXCR2 through Rho and Rac signaling pathways. Am J Physiol Lung Cell Mol Physiol 280:L1094-L1103.

Shi Y, Sawada J, Sui G, Affar el B, Whetstine JR, Lan F, Ogawa H, Luke MP, Nakatani Y (2003) Coordinated histone modifications mediated by a CtBP co-repressor complex. Nature 422:735-738.

Uhrbom L, Dai C, Celestino JC, Rosenblum MK, Fuller GN, Holland EC (2002) Ink4a-Arf loss cooperates with KRas activation in astrocytes and neural progenitors to generate glioblastomas of various morphologies depending on activated Akt. Cancer Res 62:5551-5558.

Wang H, Zhang W, Huang HJ, Liao WS, Fuller GN (2004) Analysis of the activation status of Akt, NFkappaB, and Stat3 in human diffuse gliomas. Lab Invest 84:941-951.

Weissenberger J, Loeffler S, Kappeler A, Kopf M, Lukes A, Afanasieva TA, Aguzzi A, Weis J (2004) IL-6 is required for glioma development in a mouse model. Oncogene 23:3308-3316.

White JR, Lee JM, Young PR, Hertzberg RP, Jurewicz AJ, Chaikin MA, Widdowson K, Foley JJ, Martin LD, Griswold DE, Sarau HM (1998) Identification of a potent, selective non-peptide CXCR2 antagonist that inhibits interleukin-8-induced neutrophil migration. J Biol Chem 273:10095-10098.

Yamanaka R, Tanaka R, Saitoh T, Okoshi S (1994) Cytokine gene expression on glioma cell lines and specimens. J Neurooncol 21:243-247.

Yu Z, Kone BC (2004) The STAT3 DNA-binding domain mediates interaction with NF-kappaB p65 and inducible nitric oxide synthase transrepression in mesangial cells. J Am Soc Nephrol 15:585-591.

Yu Z, Zhang W, Kone BC (2002) Signal transducers and activators of transcription 3 (STAT3) inhibits transcription of the inducible nitric oxide synthase gene by interacting with nuclear factor kappaB. Biochem J 367:97-105. 